Measur ement s of $r$ adi al prof i l e of hydrogen and deut er i um densi ty i $n$ i sot ope mixt ure pl asmas usi ng bul $k$ charge exchange spect roscopy

\begin{tabular}{|l|l|}
\hline $\begin{array}{l}\text { jour nal or } \\
\text { publ i cat i on ti l l e }\end{array}$ & Revi ew of Sci ent i f i c I nst r ument s \\
\hline vol une & 90 \\
\hline page r ange & 093503 \\
\hline year & 2019-09-06 \\
\hline URL & ht t p: //hdl . handl e. net /10655/00012542 \\
\hline
\end{tabular}




\title{
Measurements of radial profile of hydrogen and deuterium density in isotope mixture plasmas using bulk charge exchange spectroscopy
}

\author{
K.Ida* ${ }^{1,2}$ M.Yoshinuma, ${ }^{1,2}$ K.Yamasaki, ${ }^{3}$ T.Kobayashi,,${ }^{1,2}$ Y.Fujiwara, ${ }^{1}$ J.Chen, ${ }^{4}$ \\ I.Murakami, ${ }^{1,2}$ S.Satake, ${ }^{1,2}$ Y.Yamamoto, ${ }^{5}$ S.Murakami, ${ }^{5}$ and M.Kobayashi ${ }^{1,2}$ \\ ${ }^{1}$ National Institute for Fusion Science, National Institutes of Natural Sciences, Toki, Gifu 509-5292, Japan \\ ${ }^{2}$ SOKENDAI (The Graduate University for Advanced Studies), Toki, Gifu 509-5292, Japan \\ ${ }^{3}$ Research Institute for Applied Mechanics Kyushu University, Kasuga, Fukuoka, Japan \\ ${ }^{4}$ School of Nuclear Science and Technology, University of Science and Technology of China, Hefei 230026, China \\ ${ }^{5}$ Department of Nuclear Engineering, Kyoto University, Kyoto 615-8450, Japan
}

(Dated: August 27, 2019)

\begin{abstract}
Bulk charge exchange spectroscopy system has been applied to measure the radial profiles of hydrogen $(\mathrm{H})$ and deuterium $(\mathrm{D})$ density ratio in the isotope mixture plasma in Large Helical Device (LHD). Charge exchange lines of $\mathrm{H}_{\alpha}$ and $\mathrm{D}_{\alpha}$ are fitted by 4 Gaussian of $\mathrm{H}$ and $\mathrm{D}$ cold components and $\mathrm{H}$ and $\mathrm{D}$ hot components with 5 parameters by combining the measurement of plasma toroidal rotation velocity with carbon charge exchange spectroscopy. The radial profiles of relative density of hydrogen and deuterium ions are derived from $\mathrm{H}$ and $\mathrm{D}$ hot components measured and beam density calculated from beam attenuation calculation. A proof-of-principle experiment is performed by the $\mathrm{H}$ pellet and the $\mathrm{D}$ pellet injections into the $\mathrm{H}-\mathrm{D}$ mixture plasma.
\end{abstract}

PACS numbers:

\section{INTRODUCTION}

The isotope effect of ion particle transport is a very important issue in the fusion plasmas where the control of isotope ratio in the core plasma is indispensable for optimizing the fusion output due to deuterium-tritium (D$\mathrm{T})$ reaction The isotope effect of ion particle transport cannot be directly observed in the pure hydrogen $(\mathrm{H})$ or the pure deuterium (D) because of the degeneration between ion particle transport and electron particle transport due to the quasi-neutralization condition. Therefore, the transport study in the $\mathrm{D}-\mathrm{H}$ mixture plasma is essential for decoupling the ion particle transport and the electron particle transport. Recently, the difference in $\mathrm{H}$ ion transport and D ion transport in the D-H mixture plasma becomes an important topic for understanding the isotope difference in the ion transport and predicting the $\mathrm{D}-\mathrm{H}$ ratio or the $\mathrm{D}-\mathrm{T}$ ratio in the plasma.

The first measurements of transport coefficient of hydrogen isotope in tokamak plasma were performed in TFTR [1]. The tritium density is inferred from the time evolution of the neutron emissivity profiles, and transport coefficients $(\mathrm{D}, \mathrm{V})$ are determined by puffing a small amount of tritium (T) gas into deuterium plasmas. More recently, deuterium and hydrogen densities were measured in the D-H mixture plasma with a recycling particle source of $\mathrm{H}$ and $\mathrm{D}$ [2]. In these experiments, the central deuterium density and, hence, the $\mathrm{D}-\mathrm{H}$ ratio at the plasma center, are evaluated from the neutron rate,

*Electronic mail: ida@nifs.ac.jp while the $\mathrm{D}-\mathrm{H}$ ratio at the plasma edge is evaluated from the $\mathrm{H}_{\alpha}$ and $\mathrm{D}_{\alpha}$ line ratio measured with passive spectroscopy. D-H ratio profiles are found to be insensitive to the isotope species of the recycling from the wall in JET.

In spite of the importance of understanding the isotope difference in ion particle transport in the isotope mixture plasma, where the ion particle transport is decouple from electron particle transport, there are only few results reported due to a lack of precise measurement of the D-H ratio in the plasma core. In order to understand the ion particle transport in the isotope mixture plasma, a novel diagnostic technique to measure the $\mathrm{D}-\mathrm{H}$ density ratio is necessary. In this paper the measurements of the D$\mathrm{H}$ density ratio using bulk charge exchange spectroscopy combined with the measurement of carbon charge exchange spectroscopy [3] are described.

\section{EXPERIMENTAL SET UP}

In order to develop the isotope ratio measurements in the plasma core, bulk charge exchange spectroscopy system has been installed in LHD. The bulk charge exchange system consists of $300 \mathrm{~mm}$ F2.8 camera lens, 2160/mm diffraction grating, and CCD detector (Andor DU897D $16 \times 16 \mu \mathrm{m} 512 \times 512$ pixels). The dispersion is $0.72 \mathrm{~nm} / \mathrm{mm}$ at $656 \mathrm{~nm}$. 32 optical fibers with a diameter of $200 \mu \mathrm{m}$ are arranged at the entrance slit of the spectrometer, which provides the 32 channels. The time resolution is determined by the integration time of the CCD detector and is typically $5 \mathrm{~ms}$. The radial profiles of hydrogen fraction $n_{H} /\left(n_{H}+n_{D}\right)$ or deuterium fraction $n_{D} /\left(n_{H}+n_{D}\right)$ in the plasma can be measured from $\mathrm{H}_{\alpha}$ and $\mathrm{D}_{\alpha}$ lines emitted by the charge exchange reaction between the bulk ions and the neutral beam injected. Usu- 


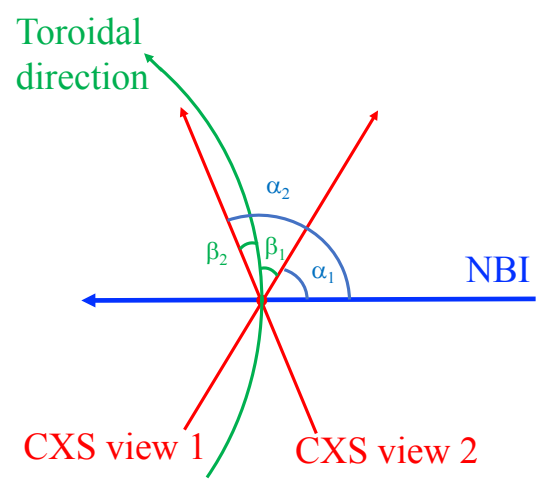

FIG. 1: Experimental set up: geometry of neutral beam, line of sight of bulk charge exchange spectroscopy.

ally, bulk charge exchange spectroscopy has been used to measure the toroidal rotation velocity in the hydrogen or deuterium plasma where the fraction of hydrogen and deuterium is close to unity and relatively constant in space $[4,5]$. In LHD, the bulk charge exchange spectroscopy [6-8] has been applied to the measurement of the radial profiles of $\mathrm{D}-\mathrm{H}$ density ratio [9] in the $\mathrm{D}-\mathrm{H}$ mixture plasma to study the isotope effect of ion particle transport.

Figure 1 shows the geometry of the line of sight, beam line, and toroidal direction in the experimental setup of LHD device. The $\alpha$ is the angle between the line of sight and beam line, while the $\beta$ is the angle between the line of sight and the toroidal direction. In order to separate the beam emission line and the charge exchange line, $\alpha$ should be small. However, the larger $\alpha$ is preferable for minimizing the error due to the uncertainty of energy dependence of the emission cross section [10]. In contrast, smaller $\beta$ is better to measure the plasma flow parallel to the magnetic flux surface, where the difference between the impurity flow and the bulk flow is relatively small compared with the flow perpendicular to the magnetic flux surface. Then the optimum of the angle between the line of sight and the beam line $\alpha_{1}$ is $60^{\circ}$ and $\alpha_{2}$ is $120^{\circ}$, while the optimum of the angle between the line of sight and the toroidal direction is $\beta_{1}=-30^{\circ}$ and $\beta_{2}=$ $30^{\circ}$. In the experimental setup in LHD, $\alpha_{1}=61^{\circ}$ and $\alpha_{2}=103^{\circ}$ and $\beta_{1}=-29^{\circ}$ and $\beta_{2}=13^{\circ}$. The angles of view 1 are close to the optimized value, while the angles of view 2 are too perpendicular to the beam line. The Doppler broadening of $\mathrm{H}_{\alpha}$ is 1.4 times larger than that of $\mathrm{D}_{\alpha}$ for the equal temperature between hydrogen and deuterium. The blue wing of the charge exchange line is mainly provided by the blue wing of $\mathrm{D}_{\alpha}(656.1 \mathrm{~nm})$, while the red wing of the charge exchange line is mainly contributed by the redwing of $\mathrm{H}_{\alpha}(656.28 \mathrm{~nm})$. Therefore, the height of the wing of the charge exchange spectra becomes asymmetric (the red wing of the spectrum is higher than the blue wing) even for the $n_{\mathrm{H}}: n_{\mathrm{D}}=1: 1$ mixture plasma without toroidal rotation. Therefore, the line of sight anti-parallel to the neutral beam line in which the beam emission is in the blue shift is preferable for avoiding the overlapping between the red wing and the beam emission.

The toroidal rotation velocity of carbon impurity, $V_{\phi}^{C}$, is derived from the Doppler shift of carbon line, $\lambda_{s}^{C}$, with the velocity correction due to the energy-dependent charge exchange cross section of carbon line, $V_{c o r}^{C}$, as

$$
V_{\phi}^{C}=c\left(\lambda_{s}^{C} / \lambda_{0}^{C}\right) / \cos \beta-\mathrm{V}_{\mathrm{cor}}^{\mathrm{C}} \cos \alpha / \cos \beta,
$$

where $\lambda_{0}^{C}$ is the wavelength of carbon line, $c$ is the velocity of light. The toroidal rotation velocity of hydrogen and deuterium, $V_{\phi}^{H}$ and $V_{\phi}^{D}$, are derived from the measured toroidal rotation velocity of carbon impurity, $V_{\phi}^{C}$, and velocity differences between hydrogen or deuterium bulk ions and carbon impurity ion calculated, $\delta V_{\phi}^{H-C}$ or $\delta V_{\phi}^{D-C}$, respectively.

$$
\begin{aligned}
& V_{\phi}^{H}=V_{\phi}^{C}+\delta V_{\phi}^{H-C}, \\
& V_{\phi}^{D}=V_{\phi}^{C}+\delta V_{\phi}^{D-C},
\end{aligned}
$$

The Doppler shift of hydrogen and deuterium, $\lambda_{s}^{H}$, and $\lambda_{s}^{D}$, are derived from the toroidal rotation velocity of hydrogen and deuterium, $V_{\phi}^{H}$ and $V_{\phi}^{D}$, with the corrections due to the energy dependent emission cross-section of hydrogen and deuterium lines, $V_{c o r}^{H}$ and $V_{c o r}^{D}$ as

$$
\begin{aligned}
& c\left(\lambda_{s}^{H} / \lambda_{0}^{H}\right)=V_{\phi}^{H} \cos \beta+\mathrm{V}_{\text {cor }}^{\mathrm{H}} \cos \alpha, \\
& c\left(\lambda_{s}^{D} / \lambda_{0}^{D}\right)=V_{\phi}^{D} \cos \beta+\mathrm{V}_{\operatorname{cor}}^{\mathrm{D}} \cos \alpha .
\end{aligned}
$$

where $\lambda_{0}^{H}$ and $\lambda_{0}^{D}$ is the wavelength of $\mathrm{H} \alpha$ and $\mathrm{D} \alpha$ lines, respectively.

\section{SPECTRUM OF H $\alpha$ AND D $\alpha$ MEASURED WITH BULK CHARGE EXCHANGE SPECTROSCOPY}

The hot component due to the active charge exchange reaction with the neutral beam is smaller than the cold component emitted in the edge by one order of magnitude. In order to subtract the cold component of the $\mathrm{H}_{\alpha}$ and $\mathrm{D}_{\alpha}$ charge exchange lines, beam modulation technique is applied. Figure 2 shows the spectra of $\mathrm{H}_{\alpha}$ and 


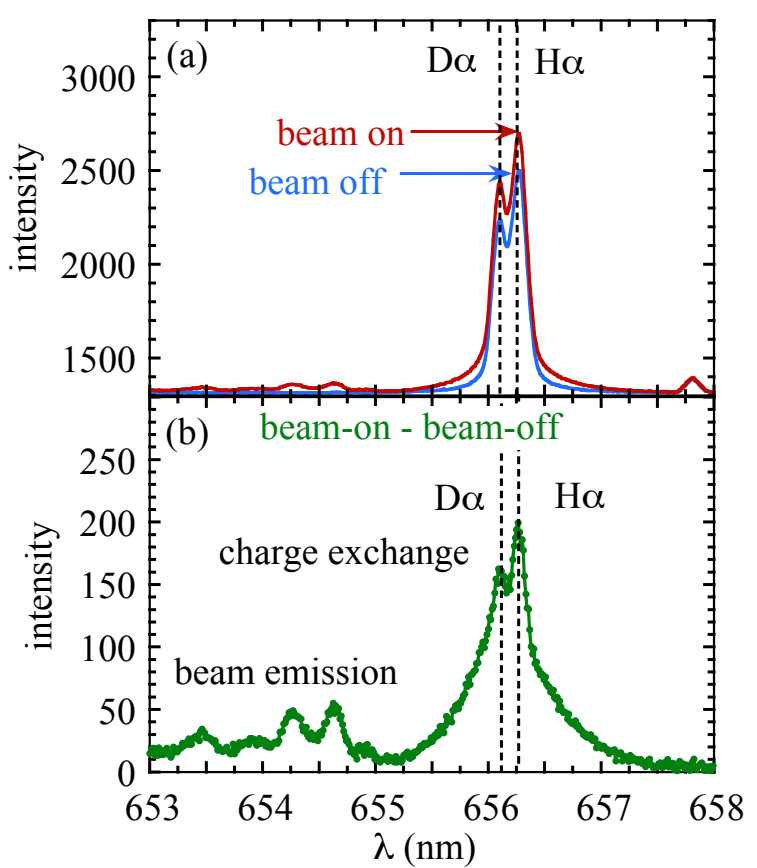

FIG. 2: $\mathrm{H} \alpha$ and $\mathrm{D} \alpha$ spectrum at (a) beam-on and beam-off timing and (b) the difference of the spectrum between beamon and beam-off timing.

$\mathrm{D}_{\alpha}$ lines at beam-on and beam-off timing and the differences in these two time slices after subtracting the spectrum at beam-off timing from the spectrum at beam-on timing for the discharge with D-H mixture plasmas. Although most of the cold components of the charge exchange lines are subtracted by the beam modulation, there still remain cold components (residual cold component) comparable to the hot components as seen in the spectrum after subtraction in the bulk charge exchange lines. There are small wings of the spectra in the plasma even without beam due to the charge exchange process between the thermal neutral penetrated into the plasma and bulk ions. This is called passive charge exchange or medium temperature component [11] and most of the medium temperature component can be subtracted by the beam modulation technique. In LHD, the radial profile of neutral is measured from this passive charge exchange component using the spectrometer with high dynamic range [12]. The passive charge exchange component is smaller than the cold component by two orders of magnitude (smaller than the active charge exchange component excited by neutral beam by a factor of 5 10). The residual passive charge exchange component is expected to be smaller than the residual cold component by two orders of magnitude (below the noise level) and can be neglected.
Because of the residual cold component, the slit width of the spectrometer is reduced to $50 \mu$ to make the instrumental width (FWHM) small enough $(0.056 \mathrm{~nm})$ to separate the two peaks of cold components of $\mathrm{H}_{\alpha}(656.28 \mathrm{~nm})$ and $\mathrm{D}_{\alpha}(656.10 \mathrm{~nm})$ lines in the spectrum. The Doppler width of the cold component is smaller than the wavelength separation $(0.18 \mathrm{~nm})$ between $\mathrm{H}_{\alpha}$ and $\mathrm{D}_{\alpha}$ lines, while the Doppler width of the hot component is too large to be separated into two peaks. The lines in the wavelength range of $653-655 \mathrm{~nm}$ are beam emissions of hydrogen beam with full, half, and one-third energy. As discussed in the previous section, the line of sight is selected to make the beam emission blue shift to have more separation between beam emission lines and charge exchange line.

\section{ANALYSIS METHOD FOR HYDROGEN AND DEUTERIUM DENSITY PROFILE MEASUREMENT}

\section{A. Fitting of spectrum with four Gaussian}

Figure 3 shows $\mathrm{H}_{\alpha}$ and $\mathrm{D}_{\alpha}$ spectra before pellet injection, after deuterium pellet injection, and after hydrogen pellet injection. The most significant differences between these two spectra appear at the red wing of the spectrum. This is because both the red shift and the wider Doppler shift of $\mathrm{H}$ hot component contribute to the increase of the red wing, while their effect cancels each other in the blue wing. Charge exchange lines are fitted by four Gaussian of $\mathrm{H}$ and $\mathrm{D}$ cold components and $\mathrm{H}$ and $\mathrm{D}$ hot components.

$$
I(\lambda)=\sum_{i}^{4}\left[A^{i} \exp \left(-\frac{\left(\lambda-\lambda_{0}^{i}-\lambda_{s}^{i}\right)^{2}}{\left(\lambda_{w}^{i}\right)^{2}+\left(\lambda_{\mathrm{ins}}\right)^{2}}\right)\right]
$$

There are 3 parameters (amplitude $A^{i}$, Doppler shift $\lambda_{s}^{i}$, and 1/e Doppler width $\lambda_{w}^{i}$ ) for each Gaussian profile and the total number of parameters for four Gaussian profiles are 12 . Here, $i$ denotes $\mathrm{H}$ and $\mathrm{D}$ hot and cold component as $i=$ Hcold, Hhot, Dcold, and Dhot. $\lambda_{0}^{i}$ is the wavelength with no Doppler shift and $\lambda_{\text {ins }}$ is the instrumental width of the spectrometer for the $50 \mu$ slit width $\left(\lambda_{0}^{\text {Hcold }}=\lambda_{0}^{\text {Hhot }}=656.28 \mathrm{~nm}\right.$, $\lambda_{0}^{\text {Dcold }}=\lambda_{0}^{\text {Dhot }}=656.1 \mathrm{~nm}$ and $\left.\lambda_{\text {ins }}=0.04 \mathrm{~nm}\right)$ In order to reduce the number of the free parameters, the Doppler width and shift of $\mathrm{H}$ and $\mathrm{D}$ cold component $\left(\lambda_{w}^{\text {Hcold }}\right.$, $\lambda_{s}^{\text {Hcold }}, \lambda_{w}^{\text {Dcold }}$ and $\lambda_{s}^{\text {Dcold }}$ ) are given by fitting the spectrum at beam-off timing with the assumption of $T_{i}^{\text {Hcold }}=$ $T_{i}^{\text {Dcold }}$. Doppler widths of H and D hot component $\left(\lambda_{w}^{\text {Hhot }}\right.$ and $\lambda_{w}^{\text {Dhot }}$ ) are given by the fitting of the spectrum after the subtraction with the assumption of equal ion temperature between hydrogen and deuterium $\left(T_{i}^{\text {Hhot }}=T_{i}^{\text {Dhot }}\right)$.

The Doppler shift of the $\mathrm{H}$ and $\mathrm{D}$ hot component $\left(\lambda_{s}^{\text {Hhot }}, \lambda_{s}^{\text {Dhot }}\right)$ is given by the toroidal carbon flow velocity with the velocity correction due to the energy- 


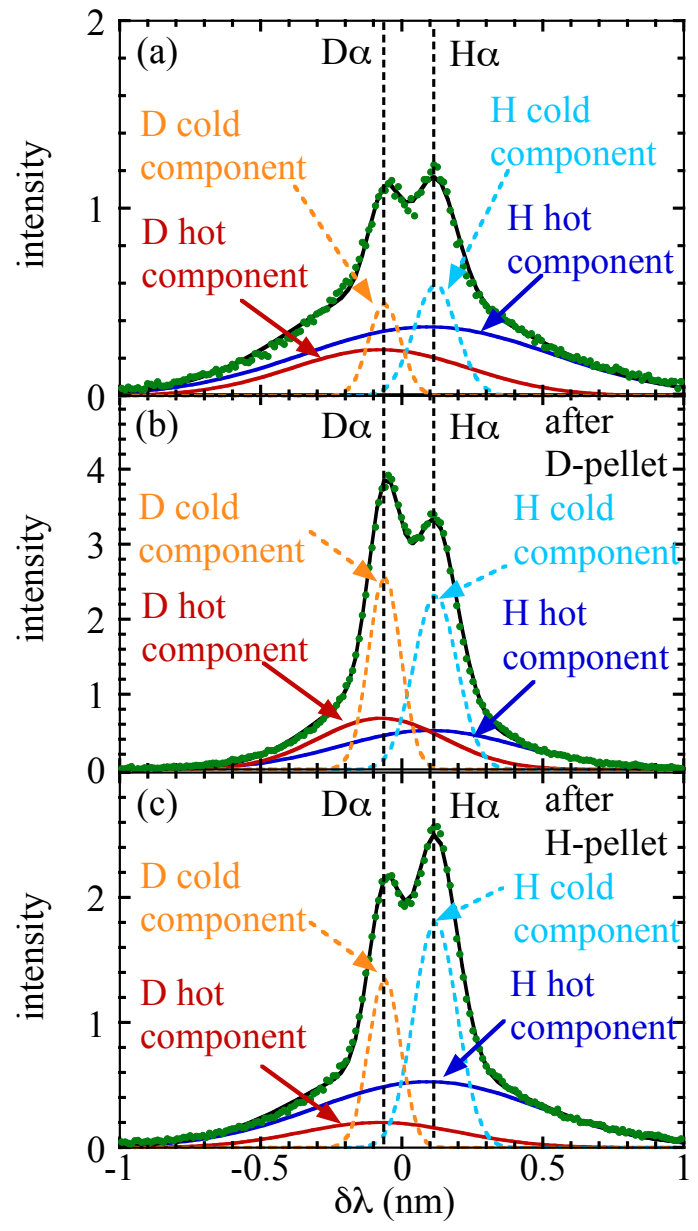

FIG. 3: $\mathrm{H} \alpha$ and $\mathrm{D} \alpha$ spectrum (a) before pellet injection, (b) after deuterium pellet injection, and (c) after hydrogen pellet injection.

dependent charge exchange cross section. Here the velocity differences between carbon and bulk ion are ignored because the difference is small enough to be neglected, as discussed in session IV-C. Then the following five free parameters for the spectrum fitting are selected: amplitude of cold and hot component of hydrogen and deuterium and ion temperature of bulk ions $\left(A^{\text {Hcold }}, A^{\text {Hhot }}\right.$, $A^{\text {Dcold }}, A^{\text {Dhot }}$, and $\left.\lambda_{w}^{\text {Hhot }}\left[216000=\sqrt{2} \lambda_{w}^{\text {Dhot }}\right]\right)$. Although both hot and cold components of hydrogen (deuterium) increase after the hydrogen (deuterium) pellet, the increase of hot component is much more significant. This is explained by the fact that the increase of the hot component is due to the increase of the density, while the increase of the cold component is due to the increase of recycling after the pellet injection.

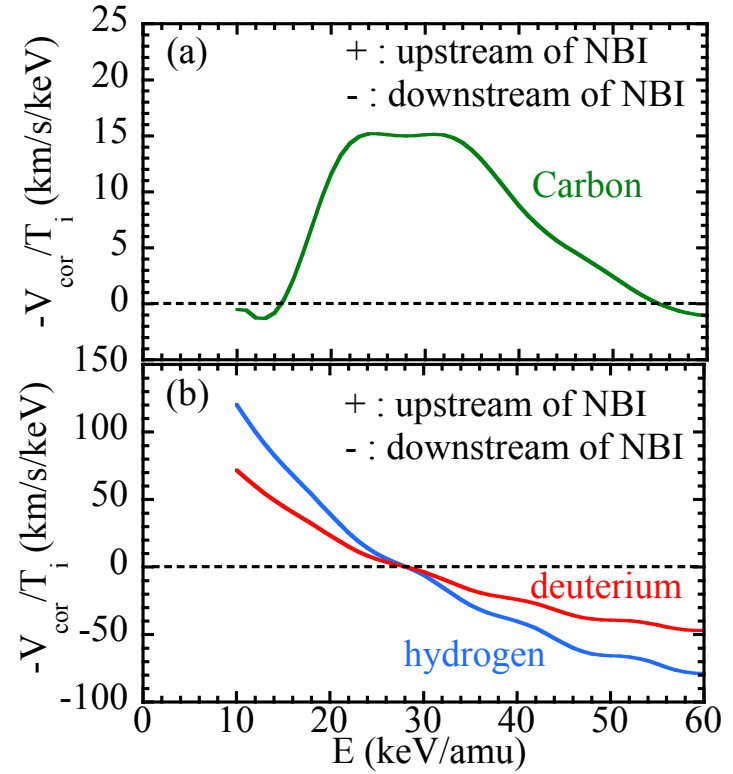

FIG. 4: Correction coefficient of toroidal velocity due to cross section effect on (a) carbon and (b) hydrogen and deuterium as a function of beam energy per atomic unit. Here the fraction of $n=2$ excited state is $0.2 \%$.

\section{B. Correction of toroidal velocity measurements}

It is well known that the rotation velocity measured with charge exchange spectroscopy requires a correction due to the energy dependent cross section because the wavelength shift appears in the charge exchange line when the $(1 / Q) \partial Q / \partial V$ is finite $[13,14]$. Here $Q$ is the emission cross section for the transition observed in the charge exchange spectroscopy and $V$ is the velocity of the probe neutral beam. The correction velocity $V_{\text {cor }}$ is roughly proportional to ion temperature and in the direction of the upstream of the neutral beam for $(1 / Q) \partial Q / \partial V>0$ and towards the downstream of the neutral beam for $(1 / Q) \partial Q / \partial V<0$. Although the correction velocity normalized by ion temperature can be evaluated by the Atomic Data and Analysis Structure (ADAS) [15], the fraction of $n=2$ excited state should be determined experimentally because the contribution of the $n$ $=2$ excited state donor to effective emission is significant at lower beam energy and has a significant influence on the values of $(1 / Q) \partial Q / \partial V$ at the beam energy below 35 $\mathrm{keV} / \mathrm{amu}$ especially for the charge exchange lines of carbon. In LHD, the $V_{\text {cor }} / T_{i}$ is experimentally determined by measuring the ion temperature dependence of wavelength shift of charge exchange line along the beam line. The $(1 / Q) \partial Q / \partial V$ evaluated from the $V_{\text {cor }} / T_{i}$ measured shows the best fit to the $(1 / Q) \partial Q / \partial V$ evaluated from ADAS with the fraction of $n=2$ excited state of $0.2 \%$.

Figure 4 shows the correction coefficient of toroidal velocity due to cross section effect for carbon and hydrogen and deuterium as a function of beam energy per atomic 


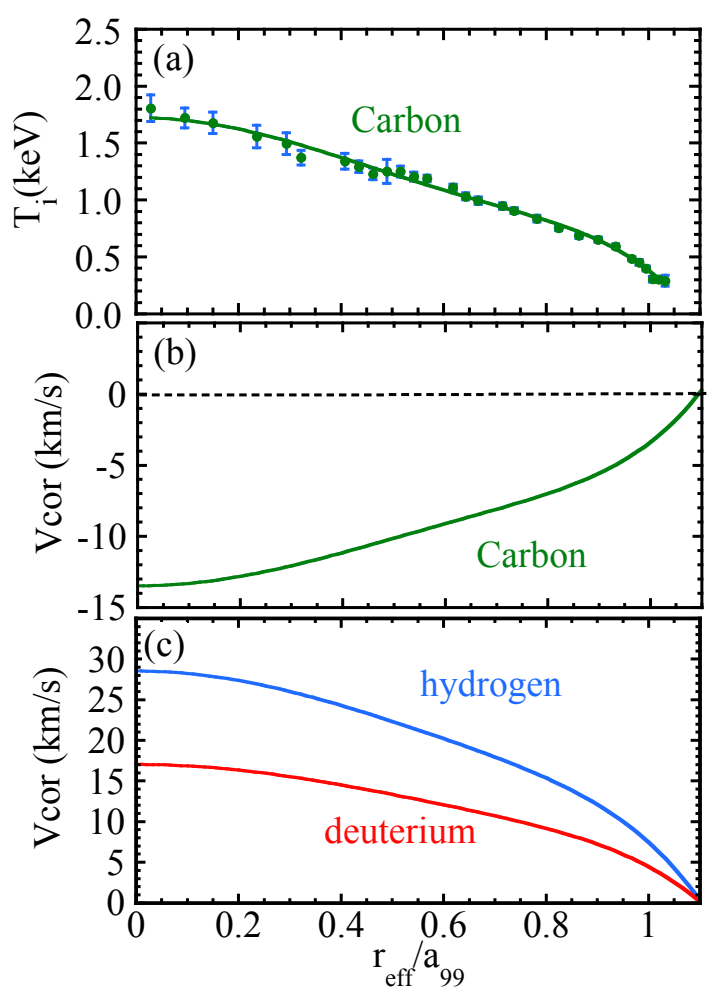

FIG. 5: Radial profile of (a) ion temperature of carbon and atomic correction of toroidal rotation for (b) carbon ion, (c) hydrogen, and deuterium ions. Here positive velocity is red shift and negative velocity is blue shift. Here the beam species is hydrogen and beam energy is $43 \mathrm{keV} / \mathrm{amu}$.

unit for the $n=2$ excited states of $0.2 \%$ [16]. In the case of the hydrogen beam where the energy range of $30 \sim 50$ $\mathrm{keV} / \mathrm{amu}$, wavelength shift due to the energy dependent charge exchange cross section is toward the upstream of the neutral beam for carbon and toward the downstream of the neutral beam for hydrogen and deuterium. However, in the case of the deuterium beam where the energy range of $20 \sim 30 \mathrm{keV} / \mathrm{amu}$, wavelength shift due to the energy dependent charge exchange cross section is toward the upstream of the neutral beam both for carbon and bulk ions. Because the velocity correction is roughly proportional to the ion temperature, precise evaluation of $(1 / Q) \partial Q / \partial V$ becomes more important at higher ion temperature. If the line of sight of the measurement is perpendicular to the beam line, the wavelength shift due to the energy dependent emission cross section can be minimized. However, in order to separate the charge exchange emission and beam emission, the line of sight should be tilted from the perpendicular direction of the neutral beam, and the optimum tilted angle to the beam line is $60^{\circ}$ in LHD.

Figure 5 shows the radial profile of ion temperature of carbon and atomic correction of toroidal rotation for carbon ion, hydrogen, and deuterium ions for the hydrogen beam with the beam energy of $43 \mathrm{keV} / \mathrm{amu}$ [16]. It should be noted that the correction of the velocity plotted in figure 5 is along the toroidal direction and not along the beam line and also not along the line of sight. Here positive velocity is red shift due to the CCW toroidal rotation and negative velocity is blue shift due to the CW toroidal rotation as seen in figure 1 . The velocity correction is $13 \mathrm{~km} / \mathrm{s}$ in $\mathrm{CW}$ direction for carbon and 17 $\mathrm{km} / \mathrm{s}$ and $28 \mathrm{~km} / \mathrm{s}$ in CCW direction for deuterium and hydrogen ion, respectively. In order to measure the D-H ratio precisely, the accurate evaluation of energy dependent emission cross section effect is essential.

\section{Velocity difference between carbon impurity and bulk ions}

In general, the flow velocity parallel to magnetic field of impurity is not equal to that of bulk ions. In LHD, the differences of flux averaged parallel flow between impurity and bulk ion $\Delta V_{i m p}$ are evaluated with neoclassical calculation using Drift Kinetic Equation Solver (DKES) code[17-19], while the differences of Pfirsch-Schlüter flow $\Delta V_{P S}$ between impurity and bulk ion are evaluated by the geometric factor $[20,21]$ with incompressibility conditions of parallel flow[22-24]. These flows are calculated from the magnetic field structure with finite pressure, and measured radial profiles of pressure and space potential. Figure 6 shows radial profile of electron temperature, electron density, radial electric field, space potential. The velocity differences between hydrogen or deuterium bulk ions and carbon impurity ion calculated by DKES code are shown in figure 6(c). The velocity difference of Pfirsch-Schlüter flow between bulk ions and carbon impurity ion calculated are also plotted. Here positive velocity is in co-direction and negative velocity is in counter-direction. The central electron temperature and the ion temperature are $3 \mathrm{keV}$, and $2 \mathrm{keV}$, respectively, while the central electron density is $3 \times 10^{19} \mathrm{~m}^{-3}$. The radial electric field is slightly positive in the core and slightly negative near the plasma periphery. As seen in figure 6 (e), the velocity differences of flux averaged parallel flow between hydrogen or deuterium bulk ions are only $2 \sim 3 \mathrm{~km} / \mathrm{s}$ and much smaller than the velocity correction. The velocity differences of Pfirsch-Schlüter flow between bulk ions are also only $2 \sim 3 \mathrm{~km} / \mathrm{s}$. (The velocity difference is identical to hydrogen and deuterium ions.) The wavelength separation between $\mathrm{H} \alpha$ and $\mathrm{D} \alpha$ is $0.18 \mathrm{~nm}$ and corresponds to the Doppler shift of bulk ions with the flow velocity of $80 \mathrm{~km} / \mathrm{s}$. Therefore, the velocity difference between carbon and bulk ion expected by the neoclassical theory $(2 \sim 3 \mathrm{~km} / \mathrm{s})$ is small enough to be neglected in this measurement in LHD. The assumption of equal toroidal flow velocity between carbon and bulk ions could be invalid in the pedestal region where the ion pressure gradient is large[25]. 


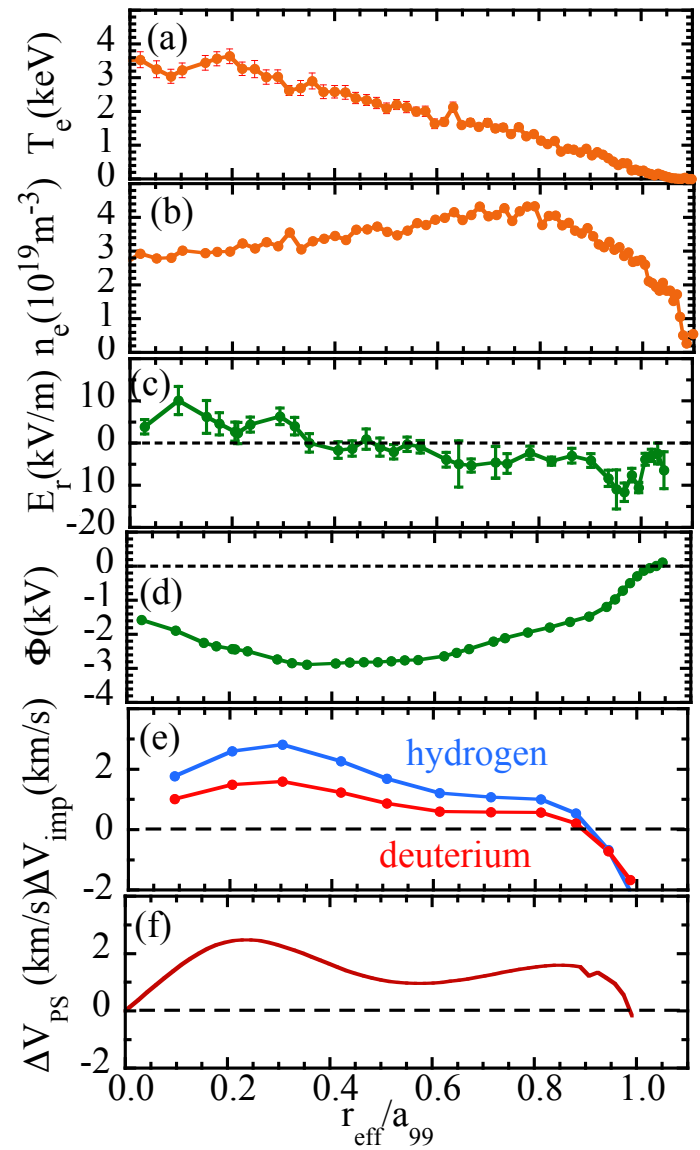

FIG. 6: Radial profile of (a) electron temperature, (b) electron density, (c) radial electric field, (d) space potential, (e) velocity differences between hydrogen or deuterium bulk ions and carbon impurity ion calculated by DKES code, and (f) velocity difference of Pfirsch-Schlüter flow between bulk ions and carbon impurity ion calculated. Here positive velocity is in co-direction and negative velocity is in counter-direction.

\section{Effect of offset of toroidal rotation velocity on the D-H ratio measurements}

Since the toroidal rotation velocity measured with carbon charge exchange spectroscopy is used to determine the wavelength shift of the $\mathrm{H}$ and $\mathrm{D}$ hot component, the uncertainty of the carbon charge exchange spectroscopy measurement has a strong influence on the determination of $\mathrm{D}-\mathrm{H}$ ratio. As seen in the toroidal ration profiles in figure $7(\mathrm{a})$, the statistical error bar of the toroidal rotation velocity measurements based on the photon noise is relatively small. The largest uncertainty in the absolute value of toroidal rotation velocity is due to the uncertainty of the absolute wavelength calibration of the spectrometer. This uncertainty of the absolute wavelength
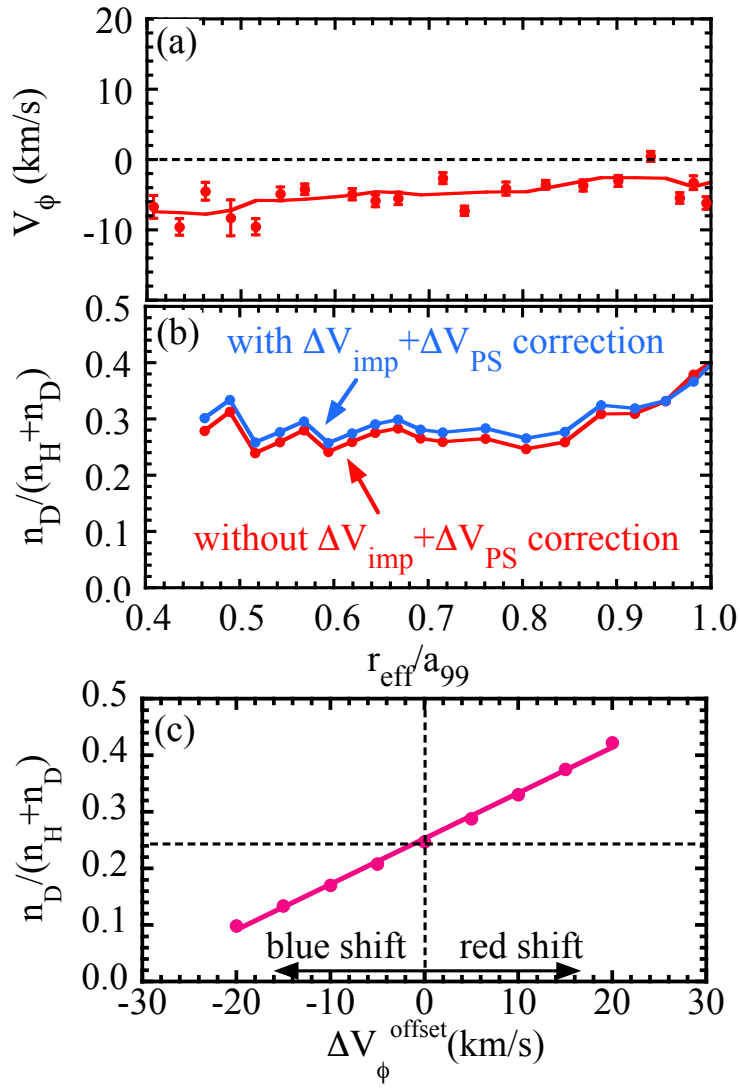

FIG. 7: Radial profile of (a) toroidal rotation velocity and (b) deuterium fraction with and without correction of velocity difference between impurity and bulk ions, and (c) the deuterium fraction as a function of the offset of carbon toroidal rotation velocity.

calibration (typically $0.01 \mathrm{~nm}$ ) gives the offset of toroidal rotation velocity of $\sim 5 \mathrm{~km} / \mathrm{s}$ in velocity. The velocity differences between carbon and bulk ions discussed in the previous section are only $2 \sim 3 \mathrm{~km} / \mathrm{s}$ and the influence of the determination of $\mathrm{D}-\mathrm{H}$ ratio is relatively small, as seen in figure $7(\mathrm{~b})$. By including the velocity differences the $\mathrm{D}$ fraction increases only $0.02 \sim 0.03$. The influence of the offset of the toroidal rotation velocity measured to the determination of $\mathrm{D}-\mathrm{H}$ ratio is studied to evaluate the systematic error bar of this measurement. The offset of the toroidal rotation is scanned from $-20 \mathrm{~km} / \mathrm{s}$ (blue shift) to $+20 \mathrm{~km} / \mathrm{s}$ (red shift). The influence is evaluated from the slope of Figure 7 (b) and is $0.008 / \mathrm{km} / \mathrm{s}$. The systematic error bar owing to the uncertainty of the offset of the carbon toroidal rotation measurement $(5 \mathrm{~km} / \mathrm{s})$ is 0.04 . 


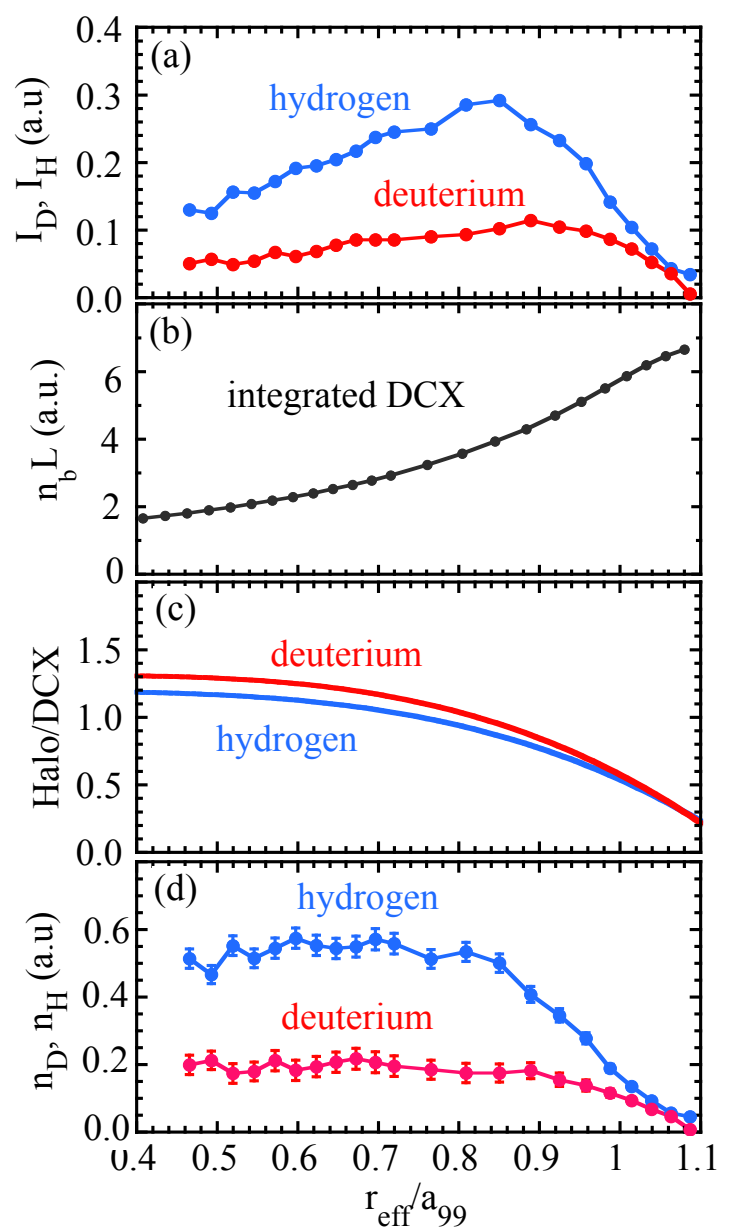

FIG. 8: Radial profile of (a) intensity of deuterium and hydrogen hot component, (b) line integrated beam density, (c) the ratio of Halo contribution to direct change exchange (DCX) contribution, and (d) density of deuterium and hydrogen.

\section{HYDROGEN AND DEUTERIUM DENSITY PROFILE IN THE D-H MIXTURE PLASMA WITH PELLET INJECTION}

Radial profiles of isotope density are calculated from the intensity of hot component and integrated beam density along the line of sight. Figure 8 shows the radial profile of intensity of deuterium and hydrogen hot component, line integrated beam density, and density of deuterium and hydrogen. The line integrated beam density is obtained by integrating the local beam density calculated by the beam attenuation code[26] based on the electron temperature and the density profiles measured. The line integrated beam density decreases toward the plasma center by a factor of $\sim 3$ due to the beam attenuation and beam divergence. In order to derive the deuterium density profile, the effect of halo neutral [27]

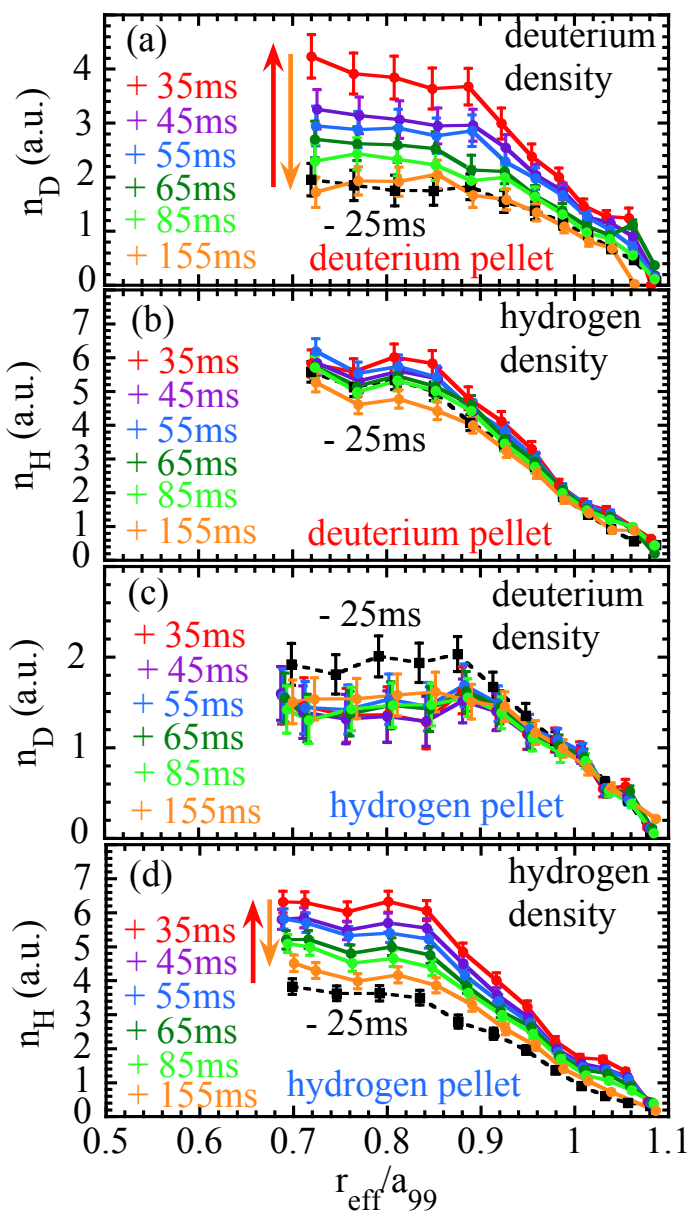

FIG. 9: Radial profile of (a)(c) deuterium and (b)(d) hydrogen density in the plasma with (a)(b) deuterium and (c)(d) hydrogen pellet injection.

should be taken into account, because the halo contribution can be very large at low temperature and high density [28]. The halo contribution is calculated with FIDAsim [29] using the density and temperature profiles measured. The difference of halo contribution by the interaction with the hydrogen ion and with the deuterium ion is relatively small as seen in Figure 8(c). Both the hydrogen and the deuterium density profiles are flat or slightly hollow in the core region and there are no significant differences in the profiles between hydrogen and deuterium.

In order to confirm the validity of this measurement, the hydrogen and deuterium pellet injection experiment into the D-H mixture plasma was performed in LHD [30]. Figure 9 shows the time evolution of radial profiles of hydrogen and deuterium density $25 \mathrm{~ms}$ before and $35 \mathrm{~ms}$ to $155 \mathrm{~ms}$ after the hydrogen and deuterium pellet injection into the hydrogen and deuterium mixture plasma. These 
results demonstrate the validity of the isotope density measurements by bulk charge exchange spectroscopy because the densities of the isotope species injected by the pellet are doubled, but the densities of the other isotope species are almost unchanged. Although ablation of the pellet is located near the plasma periphery, significant increases of ion density of the pellet are observed $35 \mathrm{~ms}$ after the pellet injection. Hydrogen and deuterium density each increase by a factor of 2 due to the particle fueling of hydrogen and deuterium pellet, respectively. Then the deuterium ion density gradually decreases to the level before the deuterium pellet injection. In contrast, hydrogen density decay is saturated at $155 \mathrm{~ms}$ after the hydrogen pellet because of the increase of the recycling of hydrogen from the wall.

\section{SUMMARY}

Bulk charge exchange spectroscopy system has been applied to measure the radial profiles of hydrogen $(\mathrm{H})$ and deuterium (D) density in the isotope mixture plasma in Large Helical Device (LHD). The line of sight of the bulk charge exchange is tilted to neutral beam by $60^{\circ}\left(90^{\circ}\right.$ is perpendicular) toward the upstream of the neutral beam in order to avoid the interference of beam emission to the red wing of $\mathrm{H}_{\alpha}$ and $\mathrm{D}_{\alpha}$ spectrum. Charge exchange lines of $\mathrm{H}_{\alpha}$ and $\mathrm{D}_{\alpha}$ are fitted by 4 Gaussian of $\mathrm{H}$ and $\mathrm{D}$ cold components and $\mathrm{H}$ and $\mathrm{D}$ hot components with 5 parameters. The measurement of plasma toroidal rotation velocity with carbon charge exchange spectroscopy are combined. Five parameters to be fitted are amplitude of cold and hot component of deuterium and hydrogen, respectively, and ion temperature of bulk ions (same ion temperature for deuterium and hydrogen ions).

The wavelength shift of hot component of $\mathrm{H}_{\alpha}$ and $\mathrm{D}_{\alpha}$ is inferred from the toroidal rotation velocity measured with carbon charge exchange spectroscopy. Although the velocity differences between carbon and bulk ions are found to be small enough to be neglected, the charge exchange cross section effect on the flow measurements are significantly large and should be taken into account using precise atomic data including the fraction of $n=2$ excited state in the neutral beam which is experimentally determined. The uncertainty of the offset of the carbon toroidal rotation velocity gives a systematic error of 0.04 in the isotope fraction in this measurement. The validity of the hydrogen and deuterium density profiles is confirmed by the hydrogen and deuterium pellet injection in the D-H mixture plasmas in LHD.

\section{ACKNOWLEDGMENTS}

The authors wish to thank the LHD Experiment Group for the excellent support of this work. One of the authors (KI) acknowledges Dr. M. Yoshida (National Institutes for Quantum and Radiological Science and Technology; QST) for providing us with two spectrometers for the bulk charge exchange spectroscopy. This work is supported by the National Institute for Fusion Science grant administrative budgets (NIFS10ULHH021,NIFS17KLPH030) and JSPS KAKENHI Grant Numbers JP15H02336, JP16H02442, and JP17H01368
[1] P.C. Efthimion, L. C. Johnson, J. D. Strachan, E.J. Synakowski, M. Zarnstorff, H. Adler, C. Barnes, R. V. Budny, F.C. Jobes, M. Louglin, D. McCune, D. Mueller, A. T. Ramsey, G. Rewoldt, A. L. Roquemore, W. M. Tang, and G. Taylor, Phys. Rev. Lett. 7585 (1995).

[2] C. Bourdelle1, Y. Camenen, J. Citrin, M. Marin, F.J. Casson, F. Koechl, M. Maslov and The JET Contributors, Nucl. Fusion 58076028 (2018).

[3] M. Yoshinuma, K.Ida, M.Yokoyama, M.Osakabe, K. Nagaoka, Fusion Sci. Technol. 58, 375 (2010).

[4] S.R. Haskey , B.A. Grierson , K.H. Burrell , C. Chrystal , R.J. Groebner, D.H. Kaplan, N. A. Pablant, and L. Stagner, Rev. Sci. Instrum. 87, 11E553 (2016).

[5] S.R. Haskey, B.A. Grierson, C. Chrystal, A. Ashourvan, K.H. Burrell, R.J. Groebner, E A Belli, L Stagner, D J Battaglia, T Stoltzfus-Dueck and A Bortolon, Plasma Phys. Control. Fusion 60105001 (2018).

[6] K. Ida, M. Yoshinuma, B. Wieland, M. Goto, Y. Nakamura, M. Kobayashi, I. Murakami, and C. Moon, Rev. Sci. Instrum. 86, 123514 (2015).

[7] K. Ida, M. Yoshinuma, M. Goto, O. Schmitz, S. Dai, A. Bader, M. Kobayashi, G. Kawamura, C. Moon, Y. Nakamura and The LHD Experiment Group, Plasma Phys.
Control. Fusion 58074010 (2016).

[8] A. Perek, K. Ida, M. Yoshinuma, M. Goto, Y. Nakamura, M. Emoto, R. Jaspers1 and LHD Experiment Group Nucl. Fusion 57076040 (2017).

[9] K. Yamazaki, K. Ida, M. Yoshinuma, T.Kobayashi, Plasma. Fusion. Res. 13, 1202103 (2018).

[10] M. von Hellermannt, P. Bregert, J. Frielingt, R. Konigt, W. Mandls, A. Maast and H.P. Summers Plasma Phy. Control. Fusion 3771 (1995).

[11] M.G. von Hellermann1, G. Bertschinger, W. Biel, C. Giroud, R. Jaspers, C. Jupen, O. Marchuk, M. OḾullane, H.P. Summers, A. Whiteford and K-D. Zastrow Physica Scripta T120 19 (2005).

[12] K. Fujii, M. Goto, S. Morita and The LHD Experiment Group Nucl. Fusion 55063029 (2015).

[13] W.M. Solomon, K.H. Burrell, P. Gohil, R.J. Groebner, L.R. Baylor, Rev. Sci. Instrum. 753481 (2004).

[14] W.M. Solomon, K.H. Burrell, R. Feder, A. Nagy, P. Gohil, R.J. Groebner, Rev. Sci. Instrum. 79 10F531 (2008).

[15] H.P. Summers, The ADAS user manual version2.6, www.adas.ac.uk, (2004).

[16] J. Chen, K. Ida, M. Yoshinuma, I. Murakami, T. Kobayashi, M.Y. Ye, B. Lyu, Phys. Lett. A 3831293 
(2019).

[17] W.I. vanRij and S.P. Hirshman, Phys. Fluids B 1563 (1989).

[18] D.A. Spong, Phys. Plasmas 12056114 (2005).

[19] H. Sugama and S. Nishimura Phys. Plasmas 15042502 (2008).

[20] V.V. Nemov, Nucl. Fusion 281727 (1988).

[21] S.T. A.Kumar, J.N. Talmadge, T.J. Dobbins, F.S.B. Anderson, K.M. Likin and D.T. Anderson, Nucl. Fusion 57 036030 (2017)

[22] H. Sugama and S. Nishimura Phys. Plasmas 94637 (2002).

[23] D.A. Spong, J.H. Harris, A.S.Ware, S.P. Hirshman and L.A. Berry, Nucl. Fusion 47626 (2007).

[24] Y.Yamamoto Phys. Plasmas, submitted.

[25] S. R. Haskey, B. A. Grierson, L. Stagner, C. Chrystal, A. Ashourvan, A. Bortolon, M. D. Boyer, K. H. Burrell,
C. Collins, R. J. Groebner, D. H. Kaplan, and N. A. Pablant, Rev. Sci. Instrum. 89, 10D110 (2018).

[26] K. Ida, M.Yoshinuma, T.Kobayashi, Y.Fujiwara, J.Chen, I.Murakami, M.Kisaki, M.Osakabe, Plasma. Fusion. Res. 141402079 (2019).

[27] R.M. McDermott, R. Dux, T. Pütterich, B. Geiger, A Kappatou, A. Lebschy, C. Bruhn, M. Cavedon., A. Frank, N. den Harder, E. Viezzer and the ASDEX Upgrade Team Plasma Phys. Control. Fusion 60095007 (2018).

[28] B.A. Grierson, K.H. Burrell, C. Chrystal, R. J. Groebner, D.H. Kaplan, W.W. Heidbrink, J. M. Muñoz Burgos, N.A. Pablant, W.M. Solomon, and M.A. Van Zeeland Rev. Sci. Instrum. 83, 10D529 (2012).

[29] W.W. Heidbrink et. al., Commun. Comput. Phys. 10716 (2011).

[30] K. Ida, et. al., Nucl. Fusion 59, 056029 (2019). 